\title{
Limb Ownership Experience and Peripersonal Space Processing
}

\author{
Robin Bekrater-Bodmann ${ }^{1}$ and Jens Foell ${ }^{2}$ \\ ${ }^{1}$ Department of Cognitive and Clinical Neuroscience, Central Institute of Mental Health, Medical Faculty Mannheim, Heidelberg University, 68159 \\ Mannheim, Germany, and 2Department of Psychology, Florida State University, Tallahassee, Florida 32306 \\ Review of Brozzoli et al.
}

The interaction of limbs and objects in peripersonal space requires the concurrent processing of their spatial relation to each other. The localization of one's own limbs by sight in relation to proximal objects involves two key regions in the brain: the posterior parietal and premotor cortices, both of which contain neurons forming bimodal receptive fields for combined visual and somatosensory input (Gentile et al., 2011). Importantly, the visual receptive fields are anchored to a specific body part, allowing adjustments for movements of both the body part and the object in peripersonal space. Until now, however, there have been no studies linking functional imaging and behavioral data with regard to limb-centered peripersonal space processing in humans, and whether limb ownership experience is an influencing factor for this process has remained an open question.

Using functional magnetic resonance imaging (fMRI), Brozzoli et al. (2012) recently conducted two experiments to close this gap of evidence. In their first experiment, they included 26 healthy right-handed subjects who were asked

Received Dec. 3, 2012; revised Dec. 20, 2012; accepted Dec. 23, 2012.

This work was supported by the PHANTOMMIND advanced grant of the European Research Council (FP7/2007-2013)/ERC Grant Agreement №. 230249.

Correspondence should be addressed to Robin Bekrater-Bodmann, Department of Cognitive and Clinical Neuroscience, Central Institute of Mental Health, Square J 5, 68159 Mannheim, Germany. E-mail: r.bekrater-bodmann@zi-mannheim.de.

DOI:10.1523/JNEUROSCI.5547-12.2013

Copyright $\odot 2013$ the authors $\quad 0270-6474 / 13 / 332729-03 \$ 15.00 / 0$ to place their right hand on a sloped table. In two separate scan sessions, the hand was placed either in their right or their left visual hemifield, while their gaze was fixed on a fixation point between both potential locations. During the scan session, an object was presented close to the right or the left position, operationalizing either a proximal or distal position of the object in relation to the participant's hand. Thus, the authors implemented a full-factorial $2 \times 2$ design to compare the neuronal responses during the processing of the position of one's hand in respect to an object which is located either proximal or distant to the visible limb.

In the second experiment, the authors used a sample of 16 healthy participants- 9 of whom had taken part in the first experiment-to investigate the importance of limb ownership experience for processing the peripersonal space by inducing the rubber hand illusion (RHI). In this setup, an artificial limb is perceived as belonging to one's own body by synchronous visuotactile stimulation together with the hidden real hand. By conducting a synchronous and an asynchronous stroking condition, Brozzoli et al. (2012) systematically manipulated the sense of ownership for the artificial hand. In the fMRI experiment, the participant's real right hand was placed covertly in the right hemifield, whereas a right rubber hand was placed visibly in the left hemifield. The partic- ipant's gaze was again fixed at a position between the real hand and the rubber hand. Participants were asked to indicate the experience of the RHI in the synchronous condition by pressing a button, at which point the experimenter presented an object near the rubber hand, as was done to the real hand in the left postural condition of the first experiment. In two subsamples of the participants, brain scans were evaluated by performing correlation analyses with self-reported vividness of the RHI or with behavioral data by assessing the socalled proprioceptive drift, which describes a shift in the perceived location of one's own hand toward the rubber hand after successful illusion induction.

By contrasting brain activity in conditions in which the object was presented in the right or the left hemifield depending on the participant's hand position, the authors found a strong interaction between hand position and the location of the object. This effect found expression in specific brain activations, especially in bilateral intraparietal sulcus (IPS) and ventral premotor cortex (PMv). Post hoc tests revealed an involvement of these key areas specifically in conditions in which the object was presented proximally to the hand. Thus, the results of the first experiment provide evidence for an involvement of premotor and parietal areas in a dynamic representation of hand-centered peripersonal space in humans. 
The RHI induction in the second experiment was successful in terms of generating significantly different values for self-reported illusion vividness as well as the proprioceptive drift data in the synchronous compared with the asynchronous stroking condition. The analyses for the corresponding functional data revealed significant stronger activity in bilateral PMv as well as in the posterior and inferior parietal areas and the insula in the synchronous stroking condition. When an object was presented near the artificial hand, the authors found stronger activity in premotor and posterior parietal areas in the synchronous stroking condition compared with the asynchronous stroking condition, which resembles the activation pattern found in the first experiment. Interestingly, they found a double dissociation between RHI measurements and activity in specific brain areas by correlating functional data with both the subjective (self-reported illusion vividness) and behavioral (proprioceptive drift) variables: there was a significant correlation between right posterior cortex and proprioceptive drift data, whereas the subjective data were significantly correlated with activity in left PMv.

These data clearly indicate a limbcentered peripersonal space processing in humans through a widely distributed frontoparietal network. Further, these results suggest not only that combined visual and proprioceptive input modulates the processing of the proximal environment, but also that the experience of limb ownership is crucial for the representational interaction of the locations of limb and object. This is indicated by the similarities between neuronal activation patterns in response to an object approaching the participant's own hand as well as an artificial limb which is perceived as belonging to the participant's body. Consequently, visual peripersonal space processing in humans does not seem to be centered at the physical body but rather at the locus of perceived body ownership. This finding might have important implications for the understanding of peripersonal space processing in humans. Thus, there is a pervasive debate whether or not tool use expands the representation of the surroundings close to the body. In particular, by reviewing and reanalyzing studies investigating a potential expansion of handcentered peripersonal space during tool use, Holmes (2012) recently challenged the notion that the published evidence supports this hypothesis. In this debate, even ownership-inducing prosthetic devices are seen as mere tools by some authors, while other authors clearly distinguish between body extensions through tool use and body extensions with accompanying ownership experiences induced by paradigms such as the RHI (De Preester and Tsakiris, 2009). The results presented by Brozzoli et al. (2012) indicate that prosthetic devices perceived as belonging to the amputee's body might change the visual representation of the proximal environment, whereas prostheses perceived as mere tools and foreign to the body might not be able to extend the peripersonal space.

However, against the background of previous studies, the question arises to what extent vision is in fact necessary for processing the peripersonal space by the stated frontoparietal network. Graziano et al. (1997) showed that receptive fields within the PMv of non-human primates still respond to an object in the peripersonal space in complete darkness, when it was seen moments ago in light. On the other hand, neurons within the IPS code for the position of a nonvisible limb (Obayashi et al., 2000), indicating that vision might play a secondary role in activating these key structures. As was recently demonstrated in congenitally blind patients, the loss of vision alters the representation of peripersonal space in the other sensory domains (Collignon et al., 2009) and diminishes the patients' ability to integrate multisensory input in a somatic RHI paradigm without visual contribution (Petkova et al., 2012). Interestingly, the experimental setup introduced by Brozzoli et al. (2012) could be easily adapted to test the importance of vision for peripersonal space processing. In analogy to the studies mentioned above, it is conceivable to modify their first experiment by intermittently removing the availability of visual information about object location in peripersonal space. In addition, the second experiment could be varied by substituting the original RHI paradigm by a somatic-only variant, which might give completely new insights in the interaction of limb ownership and peripersonal space processing.

One possible limitation of the study by Brozzoli et al. (2012) is that the maximal distance between actual hand and presented object was set to less than 30 $\mathrm{cm}$. While there is no clear or universal definition of peripersonal space, distances of up to $70 \mathrm{~cm}$ from the human body have been discussed (Holmes and Spence, 2004) and the RHI has been suc- cessfully implemented up to a distance of $30 \mathrm{~cm}$ (Lloyd, 2007). This makes it unclear whether the study design chosen by Brozzoli et al. (2012) was a true manipulation of peripersonal space representation, or whether the results might have been even more meaningful at greater distances projecting in extrapersonal space. In addition, the implementation of the RHI necessarily introduces a foreign object into the visual space around the rubber hand which applies tactile stimulation. It is unclear how this might have influenced the perception of a further object in peripersonal space, or whether the study may have benefited from using a less visually obvious stimulus than a moving paintbrush. Finally, a direct comparison of fMRI data obtained from participants who were examined in both the first (object approached the participant's real hand) and the second experiment (object approached a rubber hand that is perceived as the participant's hand) might substantiate the conclusion of the importance of ownership experiences for hand-centered peripersonal space processing.

In conclusion, Brozzoli et al. (2012) presented highly convincing data providing evidence for the integration of body-related multisensory input in peripersonal space. Their advanced design to present objects in the body's proximity with concurrent manipulation of perceived limb ownership sets new standards in the study of the visual representation of the body and its close surroundings. Thus, they presented the first data linking functional imaging of limb-centered peripersonal space processing with the sense of limb ownership, which filled a critical gap in the research of interactions between body perception and behavior in humans. This interesting new avenue of research could be advanced by prospective studies investigating the relative contribution of sensory domains in peripersonal space processing as well as examining the neuronal mechanisms of proximal environment manipulation by using tools and other body extensions with and without accompanying ownership experience.

\section{References}

Brozzoli C, Gentile G, Ehrsson HH (2012) That's near my hand! Parietal and premotor coding of hand-centered space contributes to localization and self-attribution of the hand. J Neurosci 32:14573-14582. CrossRef Medline

Collignon O, Charbonneau G, Lassonde M, 
Lepore F (2009) Early visual deprivation alters multisensory processing in peripersonal space. Neuropsychologia 47:3236-3243. CrossRef Medline

De Preester H, Tsakiris M (2009) Bodyextension versus body-incorporation: is there need for a body-model? Phenom Cogn Sci 8:307-319. CrossRef

Gentile G, Petkova VI, Ehrsson HH (2011) Integration of visual and tactile signals from the hand in the human brain: an fMRI study. J Neurophysiol 105:910-922. CrossRef Medline
Graziano MS, Hu XT, Gross CG (1997) Coding the locations of objects in the dark. Science 277:239-241. CrossRef Medline

Holmes NP (2012) Does tool use extend peripersonal space? A review and re-analysis. Exp Brain Res 218:273-282. CrossRef Medline

Holmes NP, Spence C (2004) The body schema and the multisensory representation(s) of peripersonal space. Cogn Process 5:94-105. CrossRef Medline

Lloyd DM (2007) Spatial limits on referred touch to an alien limb may reflect boundaries of visuo-tactile peripersonal space surround- ing the hand. Brain Cogn 64:104-109. CrossRef Medline

Obayashi S, Tanaka M, Iriki A (2000) Subjective image of invisible hand coded by monkey intraparietal neurons. Neuroreport 11: 3499-3505. CrossRef Medline

Petkova VI, Zetterberg H, Ehrsson HH (2012) Rubber hands feel touch, but not in blind individuals. PLoS One 7:e35912. CrossRef Medline 